\title{
Relato de caso de hidradenocarcinoma nodular
}

\author{
Case report of nodular hidradenocarcinoma
}

\section{Tiago Miranda Dias', Leonidas Braga Dias Junior², Bruno de Oliveira Santos ${ }^{3}$, Antuan Assad Iwasaka Neder ${ }^{3}$, Simone Beverly Nascimento da Costa ${ }^{4}$, Juan Andrade Guedes ${ }^{5}$, Claudia do Socorro Carvalho Miranda ${ }^{6}$, Alcinês da Silva Sousa Júnior ${ }^{7}$, Nelson Veiga Gonçalves ${ }^{8}$, Vera Regina da Cunha Menezes Palácios ${ }^{9}$}

Dias TM, Dias Junior LB, Santos BO, Neder AAI, Costa SBN, Guedes JA, Miranda CSC, Sousa Júnior AS, Gonçalves NV, Palácios VRCM. Relato de caso de hidradenocarcinoma nodular / Case report of nodular hidradenocarcinoma. Rev Med (São Paulo). 2018 set.-out.;97(4):493-7.

RESUMO: Relato de caso de hidradenocarcinoma nodular, neoplasia rara e pouco descrita na literatura, em paciente masculino, 78 anos, branco, procedente de área rural, atendido no SUS em julho de 2013, em Belém, Pará, Brasil. Foi observada lesão eritematonodular, com três meses de evolução, infiltrada, exulcerada, pouco dolorosa e sangrante, com $1 \mathrm{~cm}$ de diâmetro, na asa direita nasal, além de telangiectasias ao redor. A impressão diagnóstica foi carcinoma basocelular. A histopatologia identificou hidradenoma nodular maligno, confirmada pela imunohistoquímica. A relevância deste relato é devida a raridade da doença e a falta de protocolo para tratamento específico da mesma.

Descritores: Neoplasias; Acrospiroma/patologia; Neoplasias cutâneas/patologia.

\begin{abstract}
Case report of nodular hidradenocarcinoma, a rare neoplasm very little described in the literature, in male patient, 78 years old, caucasian, from a rural area, attended by SUS in july of 2013, Belém, Pará, Brazil. It was observed erythematododular lesion, with three months of evolution, infiltrated, exulcerated, slightly painful and bleeding, with $1 \mathrm{~cm}$ of diameter, in the right nasal wing, as well as telangiectasias around. The diagnostic impression was basal cell carcinoma. The histopathology identified malignant nodular hidradenoma, that was confirmed by immune-histochemistry. The relevance of this report is due to the rarity of the disease and the absence of protocol for specific treatment.
\end{abstract}

Keywords: Neoplasms; Acrospiroma/pathology; Skin neoplasms/ pathology.

1. Médico, residente em Patologia no Hospital A.C. Camargo Câncer Center. ORCID: 0000-0002-2905-7364. E-mail: tiagu_b@hotmail.com.

2. Médico Patologista. Professor na Universidade do Estado do Pará, Belém, PA. ORCID: 0000-0002-3549-8078. E-mail: leonidas.lbdj@gmail.com

3. Graduandos em Medicina. Universidade do Estado do Pará, Belém-PA. ORCID: Santos BO - 0000-0001-9411-4700; Neder AAI. ORCID: 00000002-9608-117X. E-mail: broliveirasantos@hotmail.com, antuanneder@hotmail.com.

4. Enfermeira. Professora Auxiliar da Universidade do Estado do Pará, Belém-PA. ORCID: 0000-0002-9817-0790, E-mail: beverlycosta@hotmail.com

5. Graduando em Engenharia Cartográfica e Agrimensura. Universidade Federal Rural da Amazônia, Belém-PA. ORCID: 0000-0003-0563-2034. E-mail: juan_andrade_guedes@hotmail.com.

6. Bióloga. Doutoranda do Programa de Pós-Graduação em Biologia Parasitária na Amazônia da Universidade do Estado do Pará, Belém-PA. ORCID 0000-0002-1913-7176. E-mail: cllaumiranda@gmail.com.

7. Professor de epidemiologia. Doutorando do Programa de Pós-Graduação em Biologia Parasitária na Amazônia da Universidade do Estado do Pará, Belém-PA. ORCID: 0000-0002-8450-6724. E-mail: alcinesjunior@gmail.com.

8. Epidemiologista, e Tecnólogo em Processamento de Dados. Professor Adjunto da Universidade Federal Rural da Amazônia, Belém-PA. ORCID: 0000-0003-0276-9318. E-mail: nelsoncg2009@gmail.com.

9. Médica Dermatologista. Professora Adjunta da Universidade do Estado do Pará, Belém-PA._ORCID: 0000-0003-0300-7972.E-mail: verareginapalacios@ gmail.com.

Endereço para correspondência: Profa. Dra. Vera Regina da Cunha Menezes Palácios. Travessa Rui Barbosa, 619-1102; Reduto, Belém, PA. CEP: 66053260. 


\section{INTRODUÇÃO}

Jo Brasil, segundo dados do Instituto Nacional do Câncer (INCA) ${ }^{1}$, em 2013 morreram 3.3126 pessoas em decorrência de câncer de pele, o que representa um aumento de $55 \%$ em 10 anos $^{2}$. Existem vários fatores de risco relacionados à ocorrência destes tipos de câncer, tais como exposição solar cumulativa, etnia branca e faixa etária ${ }^{1}$.

O hidradenocarcinoma, também conhecido como hidradenoma nodular maligno ou acrospiroma maligno, é um tumor raro com alto índice de recorrência e potencial metastático ${ }^{3}$. Esta neoplasia apresenta pouca documentação técnica e cientifica, fato este que dificulta a produção de dados epidemiológicos ${ }^{4}$.

Estudos baseados em características percentuais de casos mostram que o tumor apresenta tropismo preferencial por cabeça $(30,34 \%)$, membros superiores $(25,84 \%)$ e tronco $(20,22 \%)$. Na cabeça, o escalpe e a face são os locais mais frequentes ${ }^{5}$. Apresenta características macroscópicas de lesões nodulares de 0,2 a $5 \mathrm{~cm}$ de diâmetro com pele suprajacente normal ou rósea, podendo haver ulceração e dor a pressão ${ }^{6}$.

$\mathrm{Na}$ microscopia óptica pode ser identificada a presença de lóbulos epiteliais compostos de células com variados graus de atípia, além da desorganização da arquitetura normal, com numerosas mitoses anormais e múltiplos focos de necrose. Também podem ser observadas

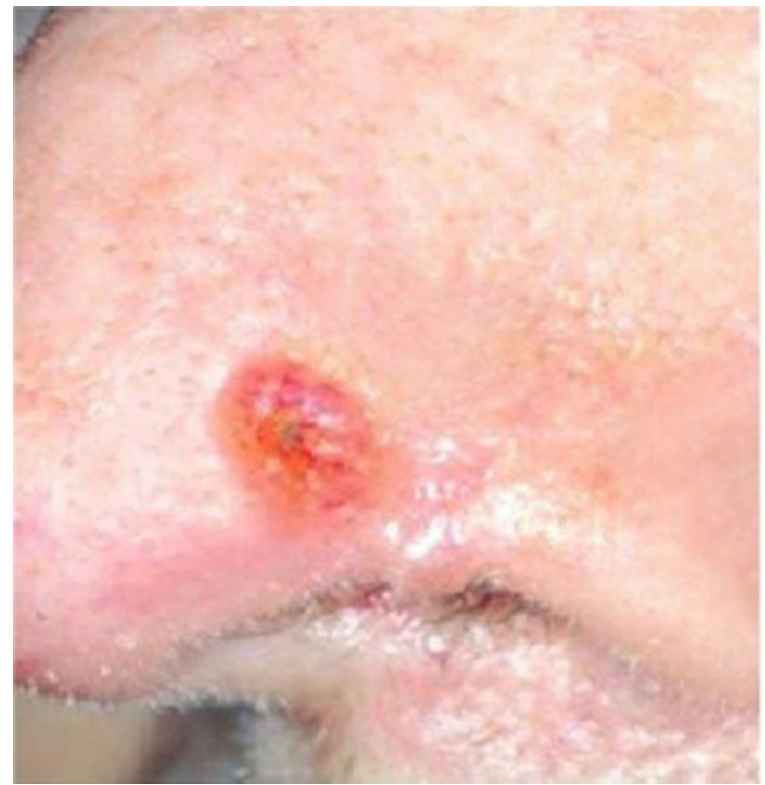

Figura 1 - Aspecto da lesão antes e depois da excisão cirúrgica

Indicada biopsia para exame histopatológico, que apresentou como resultado hidradenoma nodular maligno (HNM) (Figura 2). Após a biópsia com punch foi feita a algumas áreas com pequenos grupos de células poligonais e fusiformes eosinofílicas e células claras que contém em seu interior material PAS-positivo ${ }^{5,7}$. Os exames imuno-histoquímicos são realizados utilizando técnicas padronizadas, que envolvem o uso de anticorpos primários como: Ki-67, histona fosforilada $\mathrm{H} 3$ ( $\mathrm{PHH} 3$ ), receptor do fator de crescimento epitelial (EGFR) e Her-2/neu ${ }^{7}$.

\section{RELATO DO CASO}

O paciente acometido pela doença é faioderma, masculino, 78 anos e procedente de área rural. Ao ser atendido, em julho de 2013, apresentava lesão na asa direita do nariz, com 3 meses de evolução. Ele relatou história de exposição solar contínua, desde a adolescência, possivelmente relacionado à sua prática laboral com falta de proteção devido o desconhecimento de fatores de risco relacionados a doenças dermatológicas. O mesmo negou antecedentes oncológicos, alergias, tabagismo ou etilismo. Os exames bioquímicos de rotina que foram realizados não apresentaram alterações nos resultados.

Foi observado na ectoscopia muitas efélides distribuídas por todo o tegumento, com ceratoses principalmente nos antebraços, nas costas e na face, além de algumas exulcerações nos antebraços. A lesão nasal se apresentava eritemato-nódulo-exulcerada, infiltrada, sangrante, pouco dolorosa, de 1 x 0,8 x 0,4 cm, com aspecto macroscópico de carcinoma basocelular (Figura 1).

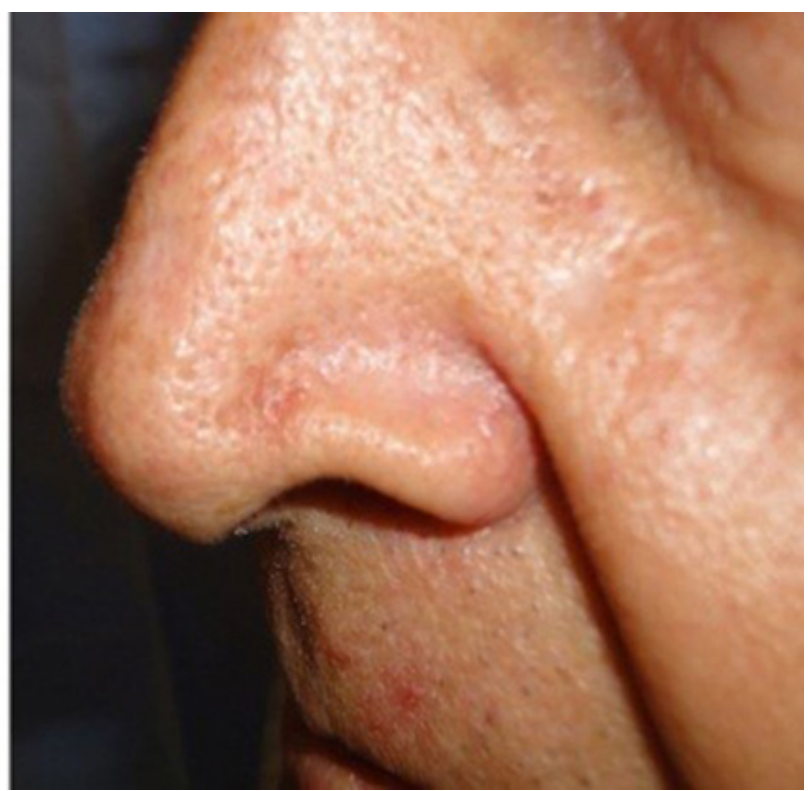

eletrocauterização até a cartilagem nasal, acreditando-se na Impressão Diagnóstica de Carcinoma Basocelular, com cicatrização por segunda intenção. 


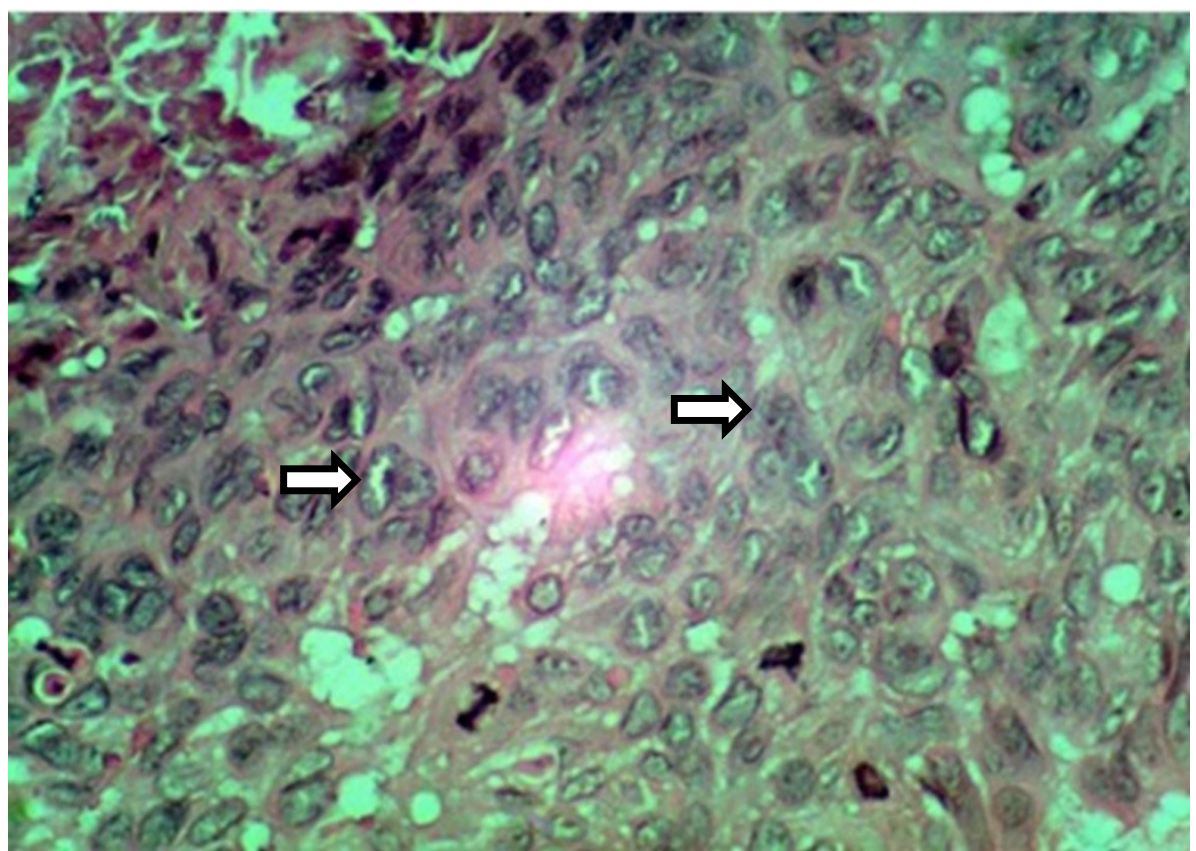

Figura 2 - Células epiteliais neoplásicas, com núcleos aumentados de volume e mitoses atípicas (H\&E, aumento original 400X)

A microscopia mostrou uma neoplasia mal delimitada, formada por proliferação de células epiteliais, com citoplasmas predominantemente amplos e claros, com núcleos vesículosos e aumentados de tamanho, cromatina granular grosseira, dispostas em arranjos sólidos, circundadas por proliferação fibrosa e infiltrado linfocitário. Focos de diferenciação escamosa e glandular e necrose central infiltrando a derme e a hipoderme, tocando as margens profunda e lateral da lesão (Figura 3).
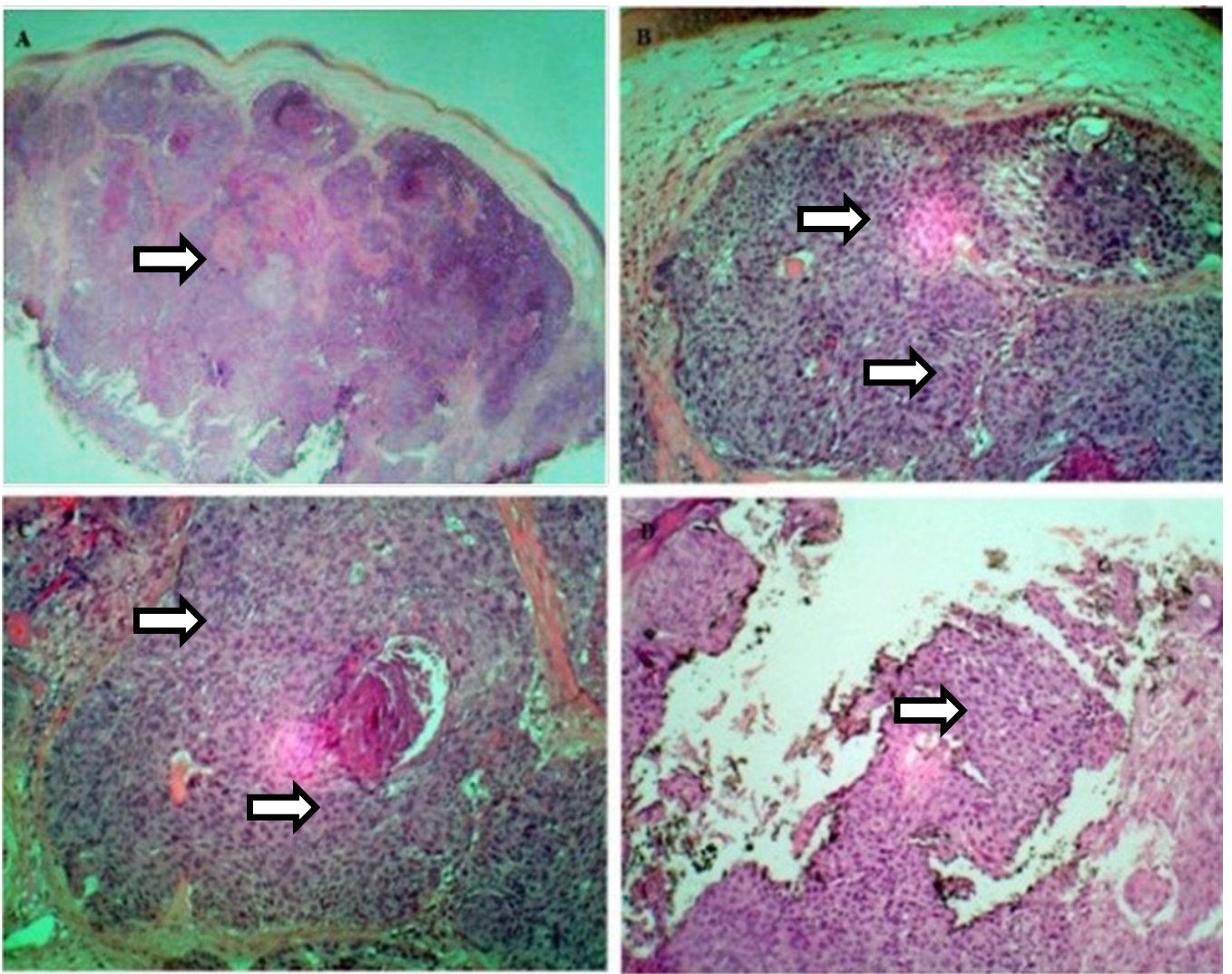

Figura 3 - Aspecto microscópico do hidradenocarcinoma. (A) Visão panorâmica de lesão nodular intradérmica se estendendo até margem lateral e profunda; (B) Grupamento sólido de células neoplásicas com foco de diferenciação glandular; (C) Grupamento sólido de células epiteliais neoplásicas com área central de necrose do tipo comedo (área necrosada); (D) Presença de células neoplásicas nas margens de ressecção, marcadas em castanho 
Após o resultado do exame histopatológico, foi indicado exame imuno-histoquímico, que além do marcador Ki-67, a lesão apresentou positividade para citoceratinas (AE1/AE3), CEA (antígeno carcino-embrionário) e vimentina de maneira focal, entretanto o EMA e a proteína S100 foram negativos. Essa tabela evidencia um alto potencial mitogênico, que corroborou com o diagnóstico ${ }^{1}$ (Figura 4).
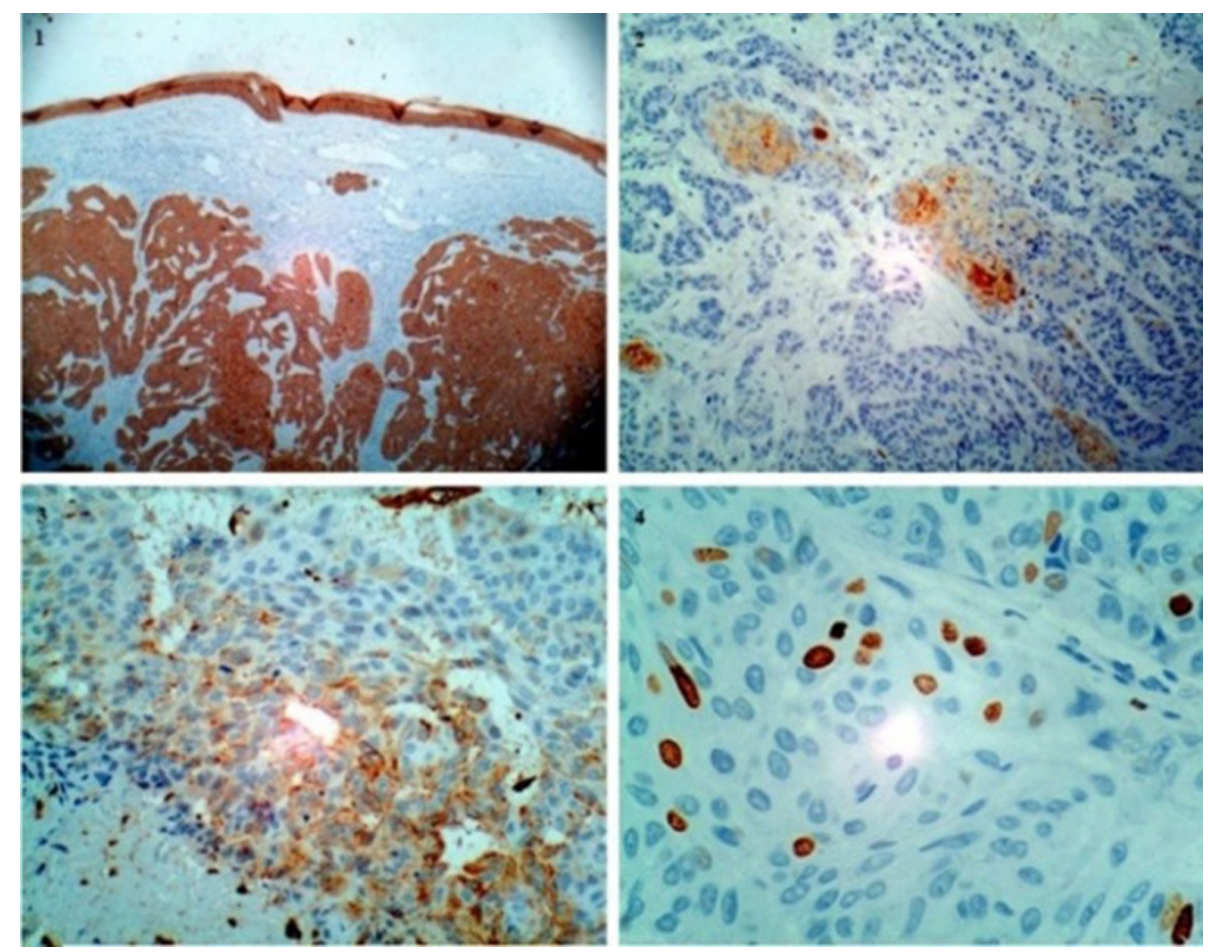

Figura 4 - Painel imuno-histoquímico para o hidradenocarcinoma. (1) Expressão das citoceratinas AE1/AE3 nas células neoplásicas; (2) Expressão de CEA em células neoplásicas; (3) Expressão citoplasmática e membranosa de Vimentina nas células neoplásicas; (4) Ki-67 expresso no núcleo das células neoplásicas

Devido à lesão apresentar potencial metastático foram realizadas tomografias computadorizadas de tórax e abdome, que não evidenciaram alterações. $\mathrm{O}$ tratamento da lesão foi prejudicado pela resistência do paciente em realizar procedimento cirúrgico mais ampliado, indicado pelo oncologista. Este fato ocorreu devido o mesmo ter concluído que estava curado. Ao longo dos últimos 4 anos, o paciente só aceita submeter-se a exames de rotina e tomografias que até o presente momento não revelam alterações, levando-nos a acreditar que a eletrocauterização tenha sido suficiente para tratar a lesão.

Desta forma, o paciente vem sendo acompanhado semestralmente pelo serviço de oncologia e dermatologia da Universidade do Estado do Pará, em Belém. Sendo que, a última consulta ocorreu em maio de 2017.

\section{DISCUSSÃO}

O perfil do paciente, objeto deste relato, ratificou a maioria dos casos de hidradenocarcinoma descritos na literatura, pois além do padrão de exposição aos fatores de risco relatados, este tipo de tumor acomete preferencialmente indivíduos acima da quinta década de vida, sendo raríssimo antes de 20 anos $^{5}$.
A localização da lesão dermatológica observada corrobora com a maioria dos casos descritos na literatura, que relatam o tropismo preferencial deste tipo de tumor pela cabeça $(30,34 \%)$, pelos membros superiores $(25,84 \%)$ e pelo tronco $(20,22 \%)$. Sendo que, na cabeça, os locais mais frequentes são no escalpe e a face ${ }^{5}$.

As características macroscópicas das lesões nodulares observadas se enquadram nos padrões descritos, na literatura, podendo variar em tamanho $(0,2$ a $5 \mathrm{~cm}$ de diâmetro) com pele suprajacente normal ou rósea, podendo ser infiltrada, com ou sem ulcerações e dor discreta a pressão $0^{5}$.

Os aspectos microscópicos apresentados nos resultados são semelhantes nas formas malignas e benignas, apresentando alguns diferenciais que caracterizam a malignidade (aumento do número de mitoses, invasão linfática ou perineural, padrões infiltrativos, áreas de necrose e padrão de crescimento disperso) ${ }^{5}$.

Considerando que a literatura descreve os hidradenocarcinomas como invasivos e de comportamento imprevisível ${ }^{10,11}$, neste trabalho as tomografias computadorizadas de tórax e abdome não mostraram alterações. Este fato concorda com outros estudos realizados $^{1,4}$, que relataram diferenças entre padrões 
relacionados às características histológicas, comportamento biológico e evolução dos casos.

Desta forma, existem relatos de casos de tumores com aspecto histológico inocente, mas com alta agressividade biológica e tumores com características de alto grau de anaplasia, que involuem sem tratamento ${ }^{1,4}$, sendo os principais sítios metastáticos os ossos, os pulmões e a pleura $^{3}$.

Financiamento: Não houve apoio financeiro.

Conflito de Interesses: Os autores declaram não haver conflito de interesses.

Agradecimentos: A Universidade Federal do Pará. Ao Laboratório de Epidemiologia e Geoprocessamento da Amazônia (EpiGeo) da Universidade do Estado do Pará. A CAPES e ao CNPq.

Participação dos autores: VRCM Palácios - trabalhou na concepção e delineamento dos dados, interpretação dos dados, redação e aprovação da versão a ser publicada; T Dias e L Dias Júnior - contribuíram com os exames histopatológicos e imunohistoquimica; BO Santos - trabalhou no delineamento e interpretação dos dados, redação e aprovação da versão a ser publicada; $A A I$ Neder - trabalhou na redação e aprovação da versão a ser publicada; SBN Costa - trabalhou na redação e aprovação da versão a ser publicada; CNSAT Martins - trabalhou na redação e aprovação da versão a ser publicada; JA Guedes - trabalhou na interpretação dos dados, redação e aprovação da versão a ser publicada; ALRR Pereira - trabalhou na redação e aprovação da versão a ser publicada; CSC Miranda - trabalhou na interpretação dos dados, redação e aprovação da versão a ser publicada; $A S$ Sousa Júnior - trabalhou na interpretação dos dados na redação e aprovação da versão a ser publicada; $N V$ Gonçalves - trabalhou no delineamento e interpretação dos dados, redação e aprovação da versão a ser publicada.

\section{REFERÊNCIAS}

1. Guimarães PV. Lesões pré-malignas em residentes em um município do Rio grande do Sul. Rev Bras Cancerol. 2014;60(3):223-30. Disponível em: http://www1.inca.gov. br/rbc/n_60/v03/pdf/06-artigo-lesoes-cutaneas-pre-malignasem-residentes-de-um-municipio-rural-do-rio-grande-do-sulbrasil.pdf.

2. Nazarian RM, Kapur P, Rakheja D, Piris A, Duncan LM, Mihm MC Jr, et al. Atypical and malignant hidroadenomas: A histological and immune-histochemical study. Mod Pathol. 2009;22(4):600-10. doi: 10.1038/modpathol.2009.18.

3. Ko CJ, Cochran AJ, Eng W, Binder SW. Hidradenocarcinoma: a histological and immunohistochemical study. J Cutan Pathol. 2006;33(11):726-30. doi: 10.1111/j.1600-0560.2006.00536.x.

4. Wong TY, Suster S, Nogita T, Duncan LM, Dickersin RG, Mihm MC Jr. Clear cell eccrine carcinomas of the skin. A clinic pathologic study of nine patients. Cancer. 1994;73(6):1631-43. doi: https://doi. org/10.1002/1097-0142(19940315)73:6<1631::AIDCNCR2820730615>3.0.CO;2-7.

5. Czernek L, Duchler M. M. Functions of cancer-derived extracellular vesicles in immunosuppressions. Arch Immunol Ther Exp. 2017;65(4):311-23. doi: 10.1007/s00005-0160453-3.

Submetido em: 01.12.17

Aceito em: 11.12.18
6. Koseki NM. Fatores associados à não-adesão ao tratamento de mulheres com câncer ginecológico ou mamário [Dissertação]. São Paulo: Universidade Estadual de Campinas (UNICAMP); 1997. Disponível em: http://repositorio.unicamp.br/jspui/ bitstream/REPOSIP/313332/1/Koseki_NancyMineko_M.pdf.

7. Machado JR AS, Vosnakis E, CruzLCP, Almeida Filho DG. Leiomioma brônquico, um raro tumor benigno. Relato de caso. J Bras Pneumol. 2000;26(3):136-9.

8. Bonadonna G, Valagussa P. Dose-response effect of adjuvant chemotherapy in breast cancer. N Engl J Med. 1981;304(1):10-5. doi: 10.1056/NEJM198101013040103

9. Escobar J, Coral G, Garcia E, Marroni CA. Hepatocarcinoma metastático pulmonar - presença de carcinomatose linfática e pulmonar. Relato de caso. J Bras Pneumol. 2000;26(3):119.

10. Oviedo RI, Ferri ÑB, Martínez BE. Hidradenocarcinoma originado en hidradenoma nodular: descripción de un caso. Rev Esp Patol. 2010;43(1):47-51. doi: 10.1016/j. patol.2009.04.001.

11. Souvatzidis P, Sbano P, Mandato F, Fimiani H, Castelli A. Castelli Malignant nodular hidradenoma of the skin: report of seven cases. J Eur Acad Dermatol Venereol. 2008;22(5):54954. doi: 10.1111/j.1468-3083.2007.02504.x. 